\title{
The intriguing plant nuclear lamina
}

\section{Malgorzata Ciska and Susana Moreno Díaz de la Espina*}

Department of Cell and Molecular Biology, Biological Research Centre - Consejo Superior de Investigaciones Científicas, Madrid, Spain

\section{Edited by: \\ Katja Graumann, Oxford Brookes \\ University, UK}

\section{Reviewed by:}

David Edgar Evans, Oxford Brookes

University, UK

Christophe Tatout, Blaise Pascal

University, France

\section{*Correspondence:}

Susana Moreno Díaz de la Espina, Department of Cell and Molecular

Biology, Biological Research CentreConsejo Superior de Investigaciones Científicas, Ramiro de Maeztu 9, 28040 Madrid, Spain

e-mail:smoreno@cib.csic.es
The nuclear lamina is a complex protein mesh attached to the inner nuclear membrane (INM), which is also associated with nuclear pore complexes. It provides mechanical support to the nucleus and nuclear envelope, and as well as facilitating the connection of the nucleoskeleton to the cytoskeleton, it is also involved in chromatin organization, gene regulation, and signaling. In metazoans, the nuclear lamina consists of a polymeric layer of lamins and other interacting proteins responsible for its association with the INM and chromatin. In plants, field emission scanning electron microscopy of nuclei, and thin section transmission electron microscopy of isolated nucleoskeletons, reveals the lamina to have a similar structure to that of metazoans. Moreover, although plants lack lamin genes and the genes encoding most lamin-binding proteins, the main functions of the lamina are fulfilled in plants. Hence, it would appear that the plant lamina is not based on lamins and that other proteins substitute for lamins in plant cells. The nuclear matrix constituent proteins are the best characterized structural proteins in the plant lamina. Although these proteins do not display strong sequence similarity to lamins, their predicted secondary structure and sub-nuclear distribution, as well as their influence on nuclear size and shape, and on heterochromatin organization, suggest they could be functional lamin analogs. In this review we shall summarize what is currently known about the organization and composition of the plant nuclear lamina and its interacting complexes, and we will discuss the activity of this structure in the plant cell and its nucleus.

Keywords: plant nuclear envelope, plant nuclear lamina, LINC proteins, NMCP proteins, CRWN proteins, SUN proteins, Nup136, plant nucleocytoplasmic linkers
The nuclear lamina is a ubiquitous structure that can be observed by transmission electron microscopy (TEM), forming a fibrous layer between the nuclear envelope (NE) and the condensed chromatin masses in many eukaryote cells, including those of protozoa and metazoans (Figure 1; Fawcett, 1966). The nuclear lamina is associated to the inner nuclear membrane (INM) and the inner side of the nuclear pore complexes (NPCs; Goldberg et al., 2008a,b; Gerace and Huber, 2012), and it is a prominent component of the nucleoskeleton (Simon and Wilson, 2011). The functions of the lamina are well established: it provides mechanical support for the nucleus and NE, it promotes the association between the nucleoskeleton and the cytoskeleton, facilitating nuclear movement and migration, and it is also involved in many activities that occur in the nucleus, such as chromatin organization and regulation and signaling (Gerace and Huber, 2012).

The metazoan lamina is a complex protein mesh that consists of a polymeric layer of lamins, intermediate filament proteins that associate with numerous transmembrane lamin-binding proteins that anchor the lamina to the INM, as well as chromatin associated factors that tether chromatin to this structure (Ho and Lammerding, 2012; Simon and Wilson, 2013). Plants contain a nuclear lamina with a similar organization to that of metazoans (Fiserova et al., 2009; Moreno Diaz de la Espina, 2009), even though plant genomes lack genes that code for lamins and lamin-binding proteins, except for the Sad1/UNC84 (SUN) domain proteins (Mans et al., 2004; Rose et al., 2004; Graumann etal., 2013) that participate in LINC (linker of the nucleoskeleton to cytoskeleton) complexes which bind the nucleoskeleton to the cytoskeleton in metazoan. In light of the crucial roles played by the lamina and by lamins in the nucleus and the cell, and given that the plant lamina is not lamin-based, many studies have focused on this structure and on the characterization of its ultrastructural and protein composition (Masuda et al., 1993, 1997; Dittmer et al., 2007; Fiserova et al., 2009; Ciska and Moreno Díaz de la Espina, 2013; Ciska et al., 2013; Sakamoto and Takagi, 2013). In this review, we use the term lamina to refer to the complex filamentous protein network associated with the INM, chromatin, nucleocytoplasmic bridging complexes, and the NPCs following the conventions applied for other eukaryotes including those that lack lamin genes. We also establish what is currently known about the structure and nature of the plant lamina, and we consider its implication in some of the activities undertaken by the metazoan lamina, such as the regulation of nuclear size and shape and chromatin organization, and also the physical connections established between the nucleoskeleton and cytoskeleton.

\section{THE METAZOAN LAMINA}

Although the first descriptions of the lamina in protozoa date from the 1950s (Pappas, 1956; Beams et al., 1957), it was not until it was described in mammalian cells that interest in the lamina became more widespread (Fawcett, 1966). Thin section TEM shows the lamina to be a thin fibrillar layer between the NE and the condensed chromatin masses (Pappas, 1956; Beams et al., 1957; Fawcett, 1966). The fibrous nature of the lamina 


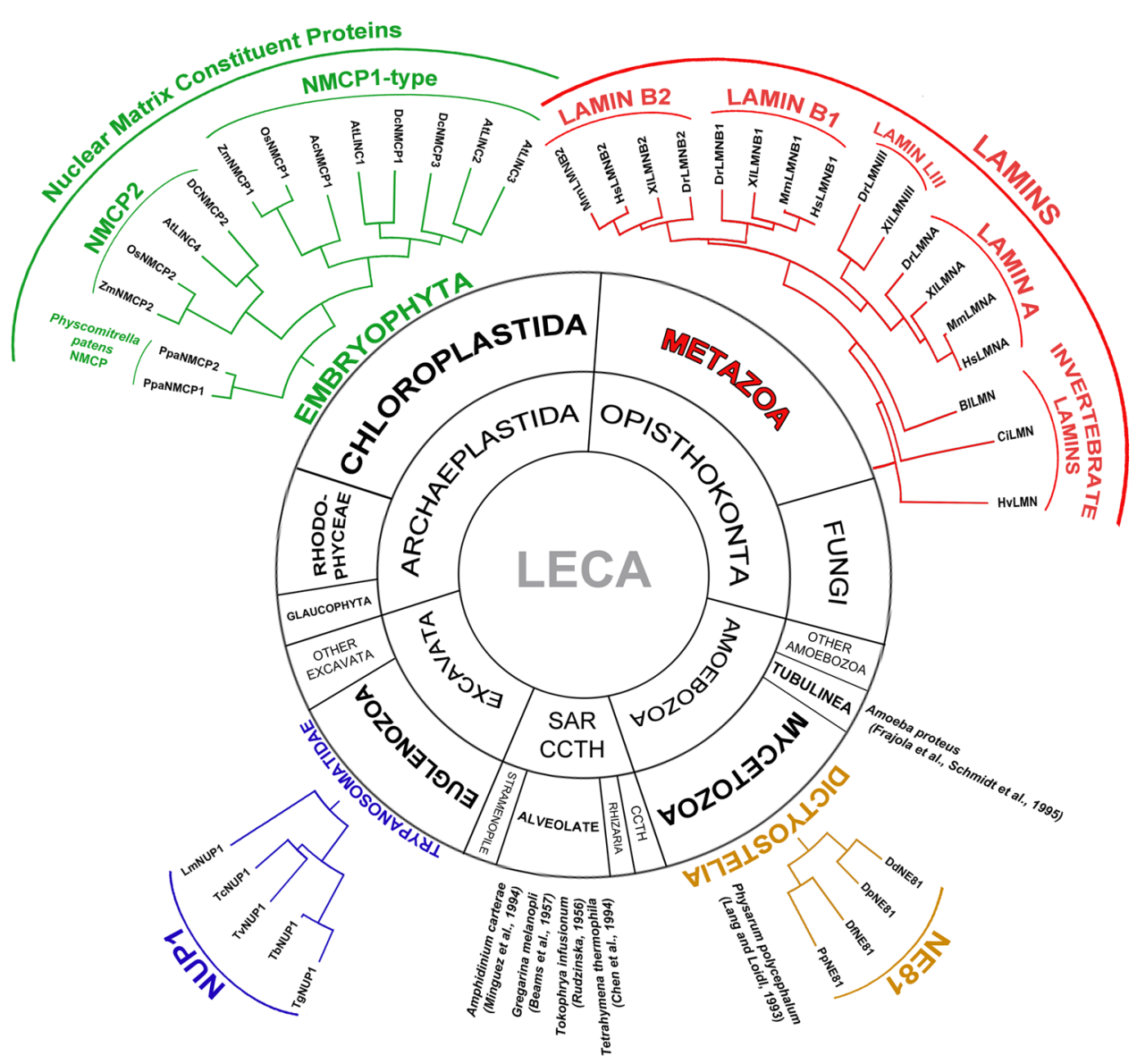

FIGURE 1 | Presence of the lamina in Eukaryota and classification and phylogenetic relationships between the lamina proteins in the different groups. The lamina has been described in different Eukaryotic groups: Metazoan, Embryophyta, Dictyostelia, Trypanosomatidae, Alveolate and Tubulinea, although its constituent proteins differ in different groups. The main components of the metazoan lamina are lamins. Most invertebrates express a single lamin while vertebrates contain four genes encoding lamin B1, lamin B2, lamin A, and lamin LIII (lost in mammals; Peter and Stick, 2012). In Dictyostelia the lamina is made up of nuclear envelope 81 (NE81) protein, which is considered an ancestor of lamins (Batsios et al., 2012; Kruger etal., 2012). The lamina was also reported in the nucleus of various Alveolate species: Amphidinium carterae, Gregarina melanopli, Tokophrya infusionum, Tetrahymena thermophila, and in Amoeba proteus (Tubulinea) and Physarum polycephalum (Dictyostelia) that do not contain a gene encoding the NE81 protein. The composition of the lamina in these species is not known. In Trypanosomatidae it is made up of a single nuclear periphery 1 (NUP1) protein (Rout and Field, 2001; Dubois et al., 2012). In Embryophyta the lamina is made up of nuclear matrix constituent proteins (NMCPs; Masuda etal., 1997; Ciska et al., 2013). NMCPs are classified in flowering plants into NMCP1-type proteins and NMCP2. Monocots have one NMCP1 and one NMCP2 proteins while dicots contain one NMCP2 and two or three NMCP1-type proteins. The moss Physcomitrella patens contains two NMCP proteins (Ciska etal., 2013). Selected species for the representation of the NMCP protein family: Allium cepa (Ac), Arabidopsis thaliana (At), Daucus carota (Dc), Oryza sativa (Os), Physcomitrella patens (Ppa), and Zea mays (Zm); NE81 proteins: Dictyostelium discoideum (Dd), Dictyostelium fasciculatum (Df), Dictyostelium purpureum (Dp), and Polysphondylium pallidum (Pp); NUP1 proteins: Trypanosoma brucei (Tb), Trypanosoma gambiense (Tg), Trypanosoma cruzi (Tc), Trypanosoma vivax (Tv), and Leishmania major (Lm); and lamins: Hydra vulgaris (Hv), Ciona intestinalis (Ci), Branchiostoma lanceolatum (BI), Danio rerio (Dr), Xenopus laevis (XI), Mus musculus (Mm), and Homo sapiens (Hs). LECA, the last eukaryotic common ancestor; SAR, stramenopile, alveolate, Rhizaria; CCTH, cryptomonads, centrohelids, telonemids, haptophytes. was corroborated when its fibrils were seen to interconnect with the NPCs in NE fractions from amphibian oocytes (Scheer et al., 1976) and rat liver cells (Aaronson and Blobel, 1975; Dwyer and Blobel, 1976). A decade later, the well organized filament network of detergent extracted NEs from Xenopus laevis oocytes was shown by TEM to have a crossover spacing of about $50 \mathrm{~nm}$ after metal shadowing (Aebi et al., 1986). Since then, the ultrastructural organization of the metazoan lamina has been characterized in amphibian oocyte NEs by feSEM (field emission scanning electron microscopy) as a regular orthogonal network of $10 \mathrm{~nm}$ filaments connected to the NPCs (Goldberg et al., 2008a,b). However, studying the filamentous network that constitutes the lamina in somatic cells is difficult due to its association with chromatin, the lamina displaying a more irregular structure in these preparations (Goldberg et al., 2008b).

The lamina was first isolated in the 1970s from rat liver nuclei, in which a conspicuous lamina could not be observed by thin section conventional TEM (Aaronson and Blobel, 1975). Its three main polypeptides were identified (Gerace et al., 1978) and later called lamins (Gerace and Blobel, 1980). Lamins are ancestral members 
of the highly conserved intermediate filament protein superfamily (Mckeon et al., 1986; Franke, 1987; Peter and Stick, 2012) and they have the same typical tripartite structure, with a central coiled coil domain formed by four coils that are separated by short linkers. Flanking the coiled coil domain, lamins have a short N-terminus that contains a conserved phosphorylation site for $c d k 1$ and a longer globular C-terminal tail with a second conserved cdk1 site, as well as a nuclear localization signal (NLS), a highly conserved Ig fold and a C-terminal cysteine, aliphatic residues, any amino acid (CAAX) box (Dechat et al., 2008, 2010; Dittmer and Misteli, 2011).

All metazoans express lamins and while invertebrates contain one or two lamin genes, there are three or four in vertebrates. Lamins have been classified as types A or B according to their structure, distribution, mitotic behavior, and biochemical characteristics (Dittmer and Misteli, 2011; Ho and Lammerding, 2012). Most invertebrates have a single type B lamin gene (Melcer et al., 2007; Peter and Stick, 2012), while the three vertebrates type B lamin genes are complemented with a forth lamin A gene that encodes lamin $\mathrm{A}$ and an alternative splicing product, lamin $\mathrm{C}$ found in mammals that have lost the gene for lamin LIII (Figure 1; Peter and Stick, 2012). Except for lamin C, all lamins are expressed as prelamins and they undergo highly regulated and extensive posttranslational modification of the CAAX box through farnesylation, proteolytic cleavage, and carboxylation. Type B lamins remain permanently modified while the 15 terminal amino acids of prelamin A are removed to produce the mature lamin A that lacks the modification (Dechat et al., 2010; Simon and Wilson, 2013). Lamins also undergo other post-translational modifications such as sumoylation and phosphorylation. Conserved phosphorylation sites for different kinases are involved in the polymerization and mitotic disassembly of lamins, as well as in the regulation of conserved functions. By contrast, unique phosphorylation sites probably mediate the differential regulation of lamins in different tissues (Simon and Wilson, 2013).

Besides lamins, the lamina contains numerous associated proteins, most of which are transmembrane proteins of the INM that bind to lamins and promote the association of the lamina with the NE. In addition, lamin-binding proteins may interact with DNA and some chromatin proteins, organizing the positioning of chromatin at the NE. Thus, lamins interact with numerous structural and regulatory proteins, many of which have mechanical and structural roles: stabilizing the lamina and anchoring lamin filaments to the INM; linking the lamina to the cytoskeleton; anchoring the lamina to NPCs; and tethering chromatin to the INM. In addition, some of these proteins regulate signaling and transcription. The lamin-binding proteins have been studied extensively (Wilson and Foisner, 2010; Ho and Lammerding, 2012; Simon and Wilson, 2013) and to date, in humans 54 binding partners have been identified for lamin A, 23 for lamin B1, and seven for lamin B2. Indeed, the functional association of many of these partners have been confirmed using molecular biology tools, including that of LEM (lamin, emerin, MAN) domain proteins, BAF (barrier to autointegration factor), Rb (retinoblastoma), and SUN domain proteins (Simon and Wilson, 2013). The partners of lamin A are involved in different nuclear activities and they include components of the nucleoskeleton and NPCs, such as lamins B1 and B2, actin, nesprin $1 \alpha$ and nesprin2, SUN1 and SUN2, nucleoporins Nup153 and Nup88, LCO1 (lamin companion 1). In addition, lamin A can associate with LEM domain proteins like LAP2a, MAN1, LEM2 and emerin, which are integral INM proteins that interact with lamins and BAF, and that form complexes involved in nuclear architecture and in anchoring chromatin to the NE. Other partners include chromatin associated proteins, such as BAF, PCNA, HP1 and histones, as well as transcription factors like $\mathrm{Rb}$ or other proteins involved in transcription and signaling (Simon and Wilson, 2013). The SUN proteins associate with Klarsicht/ANC1/syne homology (KASH) domain proteins of the outer nuclear membrane (ONM), forming the core of the LINC complex that associates with the cytoskeleton (Sosa et al., 2012; Tapley and Starr, 2013). In this way, the lamin polymer would constitute a base for the supramolecular assembly that connects the cytoskeleton with the NE and chromatin.

Although the process of lamin self assembly has been described relatively well in vitro for chicken, human and Caenorhabditis elegans lamins, the supramolecular assembly of the higher order arrays of lamins with their multiple associated proteins remains somewhat unclear due to the difficulty of reconstituting the NE environment in vitro. Lamin polymerization involves lamin dimerization, the longitudinal assembly of these dimers into oligomers that can interact laterally to form protofilaments, and the further assembly of these as $10 \mathrm{~nm}$ filaments (Ben-Harush et al., 2009; Dittmer and Misteli, 2011). The rod domains play important roles in lamin homodimerization and in the formation of lateral and longitudinal contacts (Kapinos et al., 2010; Gangemi and Degano, 2013). However, there is little information regarding how lamins are incorporated into the lamina in vivo and most of this comes from studies on the amphibian oocyte lamina. In vivo, lamins form an orthogonal mesh in the lamina connected to the inner ring of NPCs (Goldberg et al., 2008a,b; Gerace and Huber, 2012), whereby type A and B lamins form separate filament networks, although these may interact to varying degrees (Goldberg et al., 2008a,b; Kolb et al., 2011). FRAP (fluorescence recovery after photobleaching) analysis demonstrates that lamins are stably integrated into the lamina and along with lamin-associated transmembrane proteins, although the latter are more mobile than lamins (Moir et al., 2000; Ostlund et al., 2006).

The lamina is involved in many nuclear and cellular functions that are fulfilled by its multiple lamin-dependent complexes. The lamina fulfills several structural functions, regulating the size, shape, and mechanical properties of the nucleus, stabilizing the NE, positioning the NPC, mediating the physical connection between the nucleus and cytoskeleton, and positioning heterochromatin at the NE. However, it is also involved in other processes, including epigenetic modification, chromatin organization, DNA replication, repair and transcription, as well as cell proliferation, and differentiation (Dechat et al., 2008, 2009, 2010; Ho and Lammerding, 2012; Burke and Stewart, 2013).

\section{THE LAMINA IN NON-METAZOANS}

As mentioned above, the nuclear lamina is not a structure that is only found in metazoans that express lamins. A well organized 
lamina has been identified by TEM in several Protozoa species from diverse groups, including phylogenetically unrelated unicellular eukaryotes (Frajola et al., 1956; Pappas, 1956; Rudzinska, 1956; Beams et al., 1957; Lang and Loidl, 1993; Chen et al., 1994; Minguez etal., 1994), and it has also been isolated from Trypanosoma (Rout and Field, 2001; Figure 1). Except for that in Amoeba proteus and Gregarina melanopli (Frajola et al., 1956; Beams et al., 1957; Schmidt et al., 1995), the protozoan lamina resembles that of metazoans, particularly once isolated (Rout and Field, 2001). However, protozoa lack lamin orthologs and thus, their lamina is likely to be based on different proteins with similar functions. Yet to date, only two constituent proteins of the protozoan lamina that fulfill similar functions to lamin have been characterized in Dictyostelium discoideum and Trypanosoma brucei (Batsios etal., 2012; Dubois et al., 2012; Kruger et al., 2012).

Dictyostelids belong to a group of Amoebozoa that are relatively close to metazoans. The Dictyostelium lamin-like protein NE81 is restricted to the class Dictyostelia (Figure 1) and it is currently considered to be an evolutionary precursor of metazoan lamins, in particular given that it shares important structural and functional features with them, such as: size; the distribution of the coiled coils in the rod domain; the position of the cdk1 phosphorylation consensus site preceding the rod domain; the NLS in the tail; and the terminal CAAX box. Moreover, the generation of knockout and over-expression mutants has demonstrated that like lamins, NE81 plays an important role in maintaining nuclear integrity, chromatin organization, and the mechanical stability of cells (Batsios et al., 2012; Kruger et al., 2012).

Trypanosomatids are highly divergent unicellular eukaryotes and in T. brucei, NUP-1 has been shown to be the major component of the isolated lamina (Rout and Field, 2001). NUP-1 is restricted to trypanosomatids, which have a single NUP-1 ortholog (Figure 1). This is a $400 \mathrm{kDa}$ long coiled coil protein containing 20 repeats of a 144 amino acid sequence. NUP-1 is not related to lamins but it does share structural features with them, and it is also implicated in processes controlled by lamins, such as: the regulation of nuclear shape and size, the distribution of NPCs, heterochromatin organization and epigenetic control of developmentally regulated genes (Rout and Field, 2001; Dubois et al., 2012). Accordingly, the trypanosomatid lamina appears to be based on NUP-1, even though this is a protein phylogenetically unrelated to lamins.

In conjunction, the above indicates that the lamina is a ubiquitous nuclear structure with conserved functions in eukaryotes, yet the proteins that constitute the lamina in Protozoa, a group that includes phylogenetically unrelated unicellular eukaryotes, might have evolved separately from those that make up this structure in metazoans.

\section{THE PLANT LAMINA}

Although conventional thin section TEM of plant cells does not reveal a conspicuous lamina underlying the nucleoplasmic side of the NE (Figure 2A), a peripheral fibrillar layer with associated NPCs was evident in demembrated nuclei (Figure 2E), similar to the metazoan lamina, as well as in the nucleoskeleton of both monocot and dicot cells after the elimination of membranes, chromatin and soluble proteins from the nucleus (Moreno Diaz de la Espina et al., 1991; Li and Roux, 1992; Masuda et al., 1993; Minguez and Moreno Diaz de la Espina, 1993; Moreno Diaz de la Espina, 1995, 2009). Moreover, an analysis of the plant NE by feSEM confirmed the presence of a lamina similar to that of metazoans attached to the INM that was called plamina (Fiserova et al., 2009; Fiserova and Goldberg, 2010). With this technique the plant lamina appears to be a complex, organized filamentous structure that underlies the INM and that is connected to the nucleoplasmic ring of the NPCs (Figures 2B,C). Well defined tightly packed filaments were observed at the INM of tobacco cells, suggesting that the lamina would be formed by proteins that can form filaments (Figure 2C). The filaments in the lamina were $10-13$ or $5-8 \mathrm{~nm}$ thick, similar dimensions to those observed in isolated lamina fractions from pea nuclei (Li and Roux, 1992; Blumenthal et al., 2004; Fiserova et al., 2009). On view of the structural similarities of the metazoan and plant lamina, the specific term plamina recently coined for the plant lamina is not necessary, adds confusion to the field and should be avoided.

\section{PROTEIN COMPONENTS OF THE PLANT LAMINA}

Despite the structural similarities of the plant and metazoan lamina, plants lack orthologs of lamins and of the lamin-binding proteins, except for the SUN domain proteins that are conserved in all kingdoms (Mans et al., 2004; Rose et al., 2004; Moriguchi et al., 2005; Graumann et al., 2010; Murphy et al., 2010; Field et al., 2012). However, the critical functions performed by lamins and their partners in metazoan cells (see above) are fulfilled in the plant cell. Hence, the plant lamina must be established by proteins that evolved separately to those in metazoans, as is the case of the NUP-1-based lamina in trypanosomids (Figure 1; Rout and Field, 2001; Dubois et al., 2012). Similarly, such proteins would represent functional plant homologs of lamins, with similar characteristics rather than sequences. These lamin-like proteins should have a coiled coil structure similar to lamins and NUP-1, and they should be able to form filaments, to become stably integrated into the nucleoskeleton, and to participate in structural and biochemical interactions related to the formation of networks and multiprotein complexes.

Since the discovery of the plant lamina several insoluble proteins have been proposed as putative plant lamin-like proteins, mainly based on their localization in the lamina, their cross reactivity with vertebrate lamins and intermediate filaments ( $\mathrm{Li}$ and Roux, 1992; Minguez and Moreno Diaz de la Espina, 1993; Moreno Diaz de la Espina, 1995, 2009), and on their ability to form filaments in vitro (Blumenthal et al., 2004). However, the sequences of these proteins are still not available to analyze and compare them with lamins.

The best candidates to fulfill the functions of lamins in plants are the NMCPs (nuclear matrix constituent proteins) that in Arabidopsis thaliana were later called LINC (little nuclei) and very recently renamed as crowded nuclei (CRWN; Table 1), known to be components of the lamina (Figures 2D,E; Masuda etal., 1993; Ciska etal., 2013; Ciska and Moreno Díaz de la Espina, 2013; Sakamoto and Takagi, 2013). These proteins have a tripartite structure with a central coiled coil domain, and 

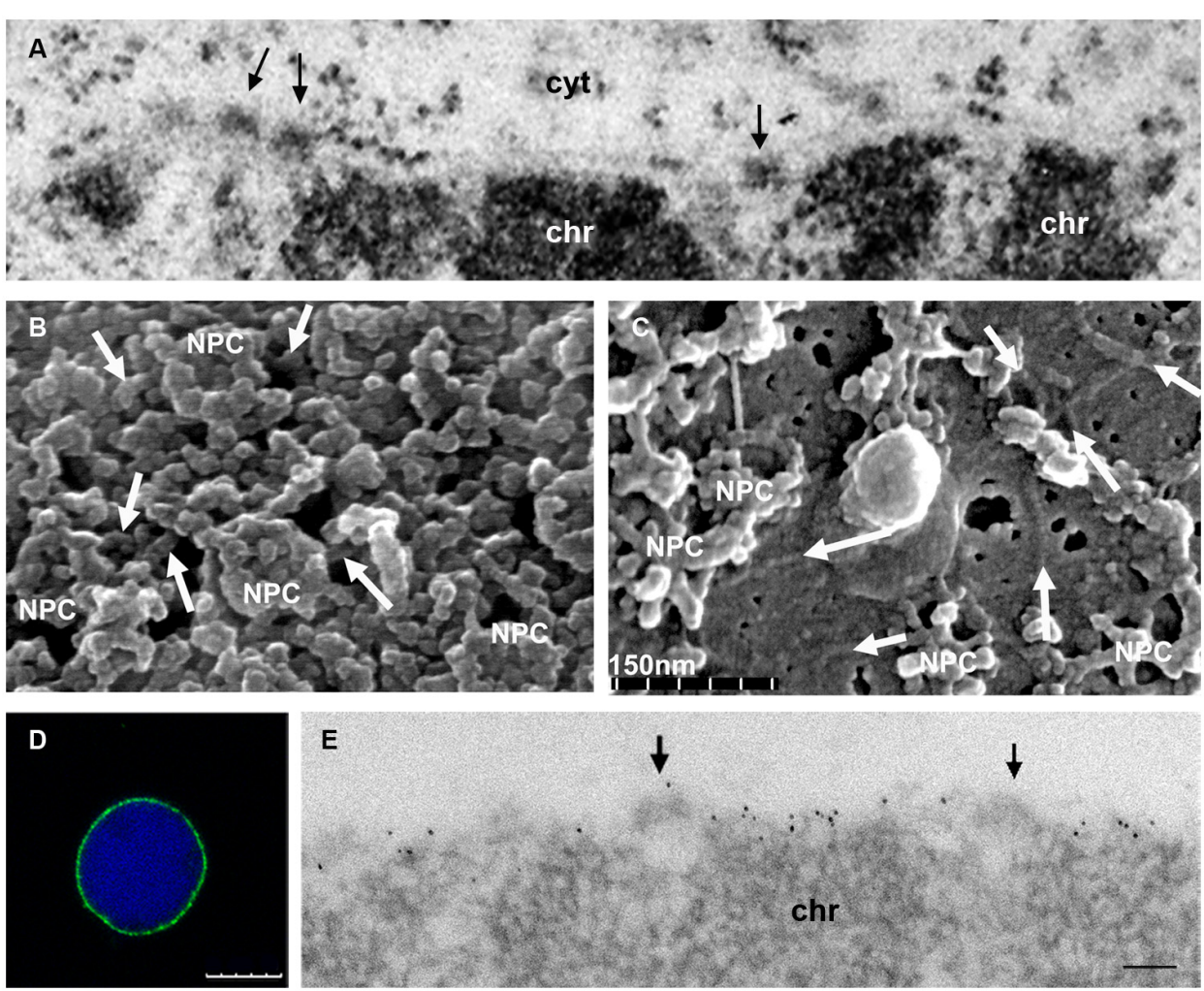

FIGURE 2 | Ultrastructure of the plant nuclear lamina and localization of the NMCP proteins. (A) Conventional thin section TEM image of the nuclear periphery of an onion meristematic root cell, showing a portion of the NE with its two membranes, and the dense NPCs (arrows) that traverse it. The heterochromatin (chr) is tightly attached to the INM but the thin lamina is not conspicuous with this technique. Cytoplasm (cyt).

(B) Cytoplasmic face of the NE of a tobacco BY-2 cell nucleus extracted with Triton $\mathrm{X}-100$ to remove the membranes and visualized by feSEM. The filaments of the lamina interconnecting the NPCs are evident (arrows). (C) feSEM image of the nucleoplasmic face of the NE of a BY-2 nucleus that has been fractured but not extracted with Triton X-100. Arrows indicate the filaments of the lamina in the membrane. (D,E) Detection of NMCP1 in the lamina of isolated meristematic onion nuclei extracted with Triton X-100 after immunofluorescence and DAPI staining (D), or TEM immunogold labeling (E). After removing the membrane, the lamina with a lower electron density than chromatin and containing NMCP1 proteins is evident at the nuclear periphery. The association with NPCs (arrows) and the tight attachment to the condensed chromatin masses (chr) can be seen. (B,C courtesy of Drs J. Fiserova and M. W. Goldberg). Bars in $D=10 \mu \mathrm{m}$ and in $E=100 \mathrm{~nm}$. they are predicted to dimerize and probably form filaments like lamins (Masuda et al., 1999; Dittmer et al., 2007; Ciska et al., 2013; Ciska and Moreno Díaz de la Espina, 2013). They also participate in the nuclear functions mediated by lamins, such as the regulation of nuclear shape and size, and heterochromatin organization (Dittmer et al., 2007; Dittmer and Richards, 2008; van Zanten etal., 2011, 2012; Sakamoto and Takagi, 2013; Wang et al., 2013). NMCPs are highly conserved in land plants (Ciska etal., 2013; Ciska and Moreno Díaz de la Espina, 2013) and although they do not share sequence similarity with lamins, their predicted structure and subnuclear distribution suggest that they participate in the formation of the plant lamina network.

\section{NMCP PROTEINS, THE PLANT ANALOGS OF LAMINS}

The first NMCP protein (DcNMCP1) was described as a residual $130 \mathrm{kDa}$ protein component of the carrot nuclear matrix (Masuda et al., 1993). The determination of its cDNA sequence enabled its structure to be predicted, similar to that of lamins with a central coiled coil domain predicted to mediate dimerization and a NLS in the tail domain (Masuda et al., 1997). Also DcNMCP1 assembled and disassembled in mitosis as occurs with lamins (Masuda et al., 1999). NMCP1 was later isolated and characterized in a monocot, Oryza sativa (Moriguchi et al., 2005). Another homolog, NMCP2, was later identified in carrot and celery (Kimura et al., 2010), and four homologs were identified in a genome-wide search for coiled coil proteins in A. thaliana (Rose et al., 2004), which were later called LINC (little nuclei) 1-4 due to the phenotype of the corresponding mutants (Dittmer et al., 2007). However, this name was misleading as it had already been attributed to the LINC complex of the NE (Crisp et al., 2006). On view of this, the same group recently renamed the proteins as CRWN according to another phenotype of the mutants (Wang et al., 2013) which adds more confusion to the nomenclature of the proteins. In our opinion the original terminology of NMCP is more appropriate not only because it was the first adopted and is currently in use for all species but A. thaliana (Table 1), but also because it refers to an intrinsic feature of the proteins. For the purposes of this review we will use the original terminology of NMCP and LINC/CRWN only for A. thaliana proteins, but the importance of the proteins and 
Table 1 | Terminology used for NMCP proteins reported in different plant species.

\begin{tabular}{|c|c|c|c|}
\hline Species & $\begin{array}{l}\text { Accession } \\
\text { number }\end{array}$ & Protein & Reference \\
\hline \multirow[t]{4}{*}{ Daucus carota (carrot) } & BAA20407 & DcNMCP1 & Masuda et al. (1993, 1997), Ciska etal. (2013), Ciska and Moreno Díaz de la Espina (2013), \\
\hline & & & Kimura et al. (2014) \\
\hline & BAl67718 & DcNMCP2 & Kimura etal. (2010), Ciska etal. (2013), Ciska and Moreno Díaz de la Espina (2013) \\
\hline & BAN14787 & DcNMCP3 & Ciska and Moreno Díaz de la Espina (2013) \\
\hline \multirow[t]{2}{*}{ Apium graveolens (celery) } & BAl67715 & AgNMCP1 & Kimura etal. (2010), Ciska et al. (2013), Ciska and Moreno Díaz de la Espina (2013) \\
\hline & BAl67716 & AgNMCP2 & Kimura etal. (2010), Ciska etal. (2013), Ciska and Moreno Díaz de la Espina (2013) \\
\hline \multirow[t]{2}{*}{ Oryza sativa (rice) } & AB110204 & OsNMCP1 & Moriguchi etal. (2005), Ciska etal. (2013), Ciska and Moreno Díaz de la Espina (2013) \\
\hline & AB110205 & OsNMCP2 & Ciska et al. (2013), Ciska and Moreno Díaz de la Espina (2013) \\
\hline Allium cepa (onion) & AB673103 & AcNMCP1 & Ciska et al. (2013), Ciska and Moreno Díaz de la Espina (2013) \\
\hline \multirow[t]{12}{*}{ Arabidopsis thaliana } & At1g67230 & NMCP1 like & Rose et al. (2004) \\
\hline & & LINC1 & Dittmer et al. (2007), Ciska etal. (2013), Sakamoto and Takagi (2013) \\
\hline & & CRWN1 & Wang et al. (2013) \\
\hline & At1g13220 & NMCP1 like & Rose et al. (2004) \\
\hline & & LINC2 & Dittmer etal. (2007), Ciska etal. (2013), Sakamoto and Takagi (2013) \\
\hline & & CRWN2 & Wang etal. (2013) \\
\hline & At1g68790 & NMCP1 like & Rose et al. (2004) \\
\hline & & LINC3 & Dittmer et al. (2007), Ciska etal. (2013), Sakamoto and Takagi (2013) \\
\hline & & CRWN3 & Wang et al. (2013) \\
\hline & At5g65770 & NMCP1 like & Rose et al. (2004) \\
\hline & & LINC4 & Dittmer etal. (2007), Ciska etal. (2013), Sakamoto and Takagi (2013) \\
\hline & & CRWN4 & Wang etal. (2013) \\
\hline
\end{tabular}

the expected future development of the field deserve an agreement of the different groups involved to establish a common terminology.

Recent searches of plant genomes have revealed that NMCP genes are conserved in land plants, which contain genes coding for two or more NMCP proteins (Kimura etal., 2010; Ciska etal., 2013; Ciska and Moreno Díaz de la Espina, 2013). NMCPs constitute a highly conserved family of proteins in plants, except in single cell plants, and they are absent from metazoans and fungi (Ciska et al., 2013; Ciska and Moreno Díaz de la Espina, 2013). NMCP proteins have been classified into two clusters following their first denominations: NMCP1 and NMCP2 (Figure 1; Masuda et al., 1993, 1997; Kimura et al., 2010; Ciska et al., 2013; Ciska and Moreno Díaz de la Espina, 2013; Wang et al., 2013). Monocots have one NMCP1 and one NMCP2 gene, while dicots carry a single NMCP2 gene and several NMCP1type genes encoding NMCP1-related proteins called NMCP1 and NMCP3. A. thaliana and some other dicots contain two NMCP3type genes, while two Solanum species, Solanum tuberosum and $S$. lycopersicum have two NMCP1 genes and no genes encoding NMCP3. AtLINC1/CRWN1 encodes an NMCP1, whereas AtLINC2/CRWN2 and AtLINC3/CRWN3 encode NMCP3-type and AtLINC4/CRWN4 encodes an NMCP2 protein (Ciska et al., 2013; Ciska and Moreno Díaz de la Espina, 2013; Wang etal., 2013). The two NMCP genes of the moss Physcomitrella patens evolved from a common NMCP progenitor gene and they are included in the NMCP2 cluster, suggesting that the archetypal NMCP progenitor was actually an NMCP2 protein (Ciska et al., 2013; Ciska and Moreno Díaz de la Espina, 2013). All LINC/CRWN genes are expressed in whole $A$. thaliana plants (Sakamoto and Takagi, 2013) and their expression is developmentally regulated (Ciska et al., 2013; Ciska and Moreno Díaz de la Espina, 2013), as occurs with lamins (Benavente et al., 1985; Broers et al., 1997; Peter and Stick, 2012).

The predicted structure of NMCP proteins is well characterized (Figure 3; Ciska et al., 2013; Ciska and Moreno Díaz de la Espina, 2013). As indicated above, they have a tripartite structure similar to that of lamins with a highly conserved central coiled coil rod domain that is predicted to dimerize, and less conserved noncoiled coil head and tail domains. The structure and length of the rod domain are conserved across the NMCP family, suggesting that it plays an important role in oligomerization. Moreover, at each end of the rod domain of NMCPs and in the predicted linkers there are five highly conserved and family specific regions. Lamins have a similar distribution of conserved motifs although there is no significant sequence similarity between these two protein families (Figure 3). NMCPs also contain several conserved motifs in the tail domain, including a stretch of acidic amino acids that is also present in vertebrate lamins, a NLS and a NLS-linked conserved motif in NMCP1's, both necessary for association of the protein 


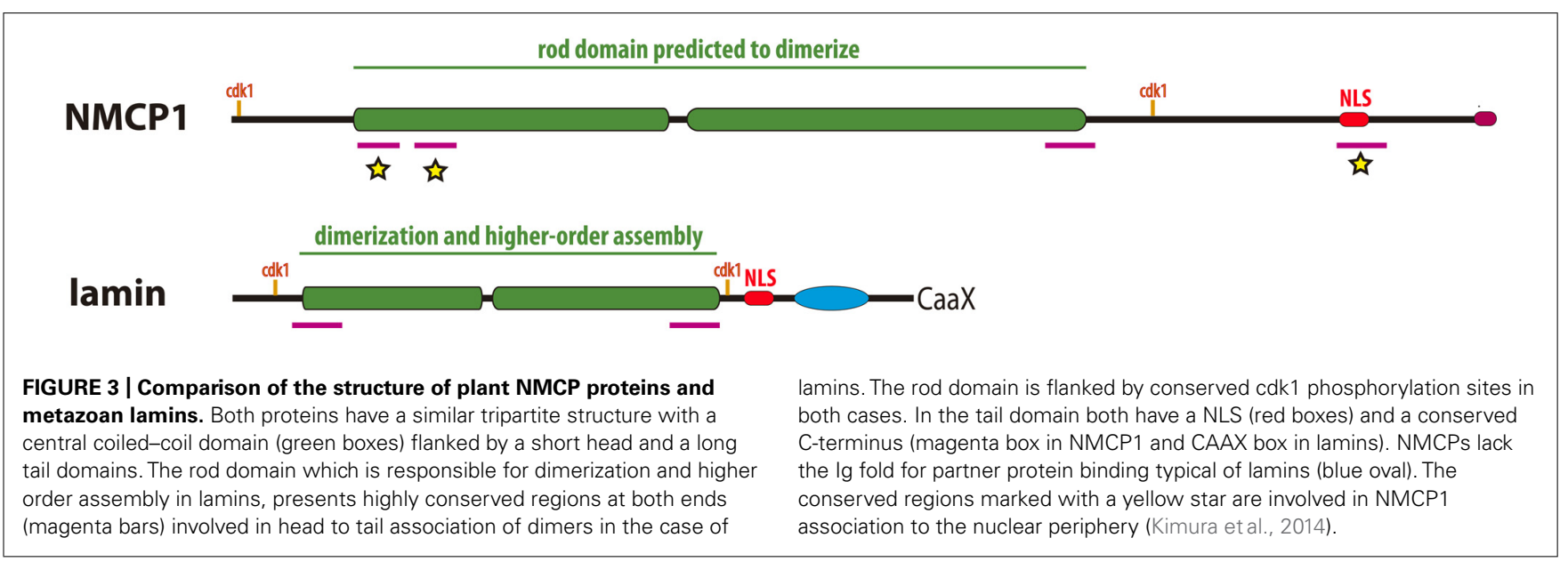

to the NE (Kimura et al., 2014). The later is identical to a specific actin binding site in lamin A. NMCPs lack a CAAX box but their Cterminus is highly conserved, except for that in the dicot NMCP2 (Ciska et al., 2013). They also lack the Ig fold in the tail that is involved in the interaction of lamins with some of their protein partners (Simon and Wilson, 2013).

The general organization of the rod domain in NMCPs and lamins is similar, although the former is twice as long as that in lamins, with a similar distribution of conserved motifs, including those at their ends (Ciska and Moreno Díaz de la Espina, 2013) that mediate head to tail associations in lamins (Kapinos et al., 2010; Davidson and Lammerding, 2013). The analogous structures, the similar location of the conserved motifs in the rod domain and the presence of consensus sequences for cdk1 at either side of the rod domain, suggest a similar mechanism of oligomerization and protofilament formation for NMCPs and lamins (Figure 3). As yet, NMCP proteins have not been polymerized in vitro and their mechanisms of assembly in the lamina are poorly known. Very recently it has been established that DcNMCP1 associates to the nuclear periphery by coordinate action of the NLS and a NLS-linked conserved motif and the 141 amino acids at the $\mathrm{N}$-terminus of the protein comprising the head and the highly conserved N-terminus of the rod domain (Kimura et al., 2014; see Figure 3). The N-terminal region of NMCP1 could be involved in head to tail assembly of dimers and polymer assembly stabilization as occurs in lamins (Davidson and Lammerding, 2013) and may be critical for the integration of the proteins in the lamina network. The immuno-feSEM experiments that are in progress should help to detect the NMCP1 protein in the filaments of the plant lamina.

The functions of NMCP proteins are poorly understood as the phenotypic effects of their mutations are not as severe as those caused by mutations in lamins (Butin-Israeli et al., 2012; Ho and Lammerding, 2012). NMCP proteins are involved in essential processes as quadruple NMCP/LINC/CRWN mutants are not viable. By contrast, single, double and some triple mutants are viable (Dittmer etal., 2007; Sakamoto and Takagi, 2013; Wang et al., 2013), which in conjunction with the lack of phenotype of single mutants, indicates that there is complementation between different proteins.
To date, the best analyzed function of NMCP/LINC/CRWN proteins is the regulation of nuclear size and shape (Dittmer et al., 2007; van Zanten et al., 2011; Sakamoto and Takagi, 2013; Wang et al., 2013), a function also fulfilled by lamins (Ho and Lammerding, 2012). NMCP/LINC/CRWN mutations result in a decrease in nuclear size and alterations to the shape of differentiated cells, with a predominant influence of the LINC1/CRWN1 and LINC4/CRWN4 genes (Dittmer et al., 2007; Dittmer and Richards, 2008; van Zanten etal., 2011; Sakamoto and Takagi, 2013; Wang et al., 2013). In addition, over-expression of LINC4 results in an increase in nuclear size (Sakamoto and Takagi, 2013), although the underlying molecular mechanisms involved remain unknown. Other proteins of the plant NE that also affect nuclear size and shape are those forming the nucleocytoplasmic linker in plants, such as the SUN domain proteins (Oda and Fukuda, 2011; Zhou et al., 2012), the KASH-like WIP (WPP domain interacting proteins) proteins (Zhou et al., 2012) and the WIT (WPP domain-interacting tail-anchored) proteins (Tamura et al., 2013), as well as nucleoporin Nup136 (Tamura et al., 2010; Tamura and Hara-Nishimura, 2011). These results suggest that the proteins forming the plant nucleocytoplasmic linker interact with NMCPs, as well as Nup136, a component of the nucleoplasmic basket of NPCs that has been proposed to link the NPC to the lamina in plants (Tamura and HaraNishimura, 2013), thereby cooperating in the regulation of nuclear morphology.

The role of NMCP proteins in chromatin organization remains unclear. A decrease in the number of chromocentres was reported in linc1/crwn1-linc2/crwn2 mutants (Dittmer etal., 2007), although the relative heterochromatin fraction and the distribution of specific heterochromatin regions during seed germination was unaltered in these mutants (van Zanten etal., 2011, 2012). Nevertheless very recent results using different mutants showed that chromocentre organization is disrupted in linc4/crwn4 mutants, as demonstrated by the dispersion of 5S RNA genes and centromeric repeat arrays (Wang etal., 2013). Accordingly, it was suggested that $\mathrm{NMCP} / \mathrm{LINC/CRWN}$ proteins play a role in maintaining proper heterochromatin organization. Thus, NMCP1/LINC1/CRWN1 and NMCP3/LINC2/CRWN2 could prevent chromocentre 
aggregation while NMCP2/LINC4/CRWN4 would have a complementary role in maintaining chromocentre integrity (Wang et al., 2013).

Lamins mediate nuclear positioning and movement through an interaction between lamin A with SUN proteins, which associate with KASH proteins to form the metazoan LINC complex that connects the lamina to the cytoskeleton (Sosa et al., 2012; Tapley and Starr, 2013). Very recently, a nucleocytoplasmic linker has been described in plants, which is involved in nuclear positioning and movement in response to environmental stimuli. It consists of a plant-specific Myosin Xl-i motor that binds to the actin filaments of the cytoskeleton, and also to the WIT proteins in the ONM that form a complex with WIP proteins, in turn interacting with SUN proteins (Tamura et al., 2013). Thus, the complex formed by the WIT, WIP, and SUN proteins in plants would be analogous to the metazoan LINC complex. While connections between this complex and NMCP proteins (or other intranuclear components) are yet to be defined, an interaction between NMCP and SUN has been recently demonstrated (Graumann, 2014). The analysis of linc/crwn1-4 and linc/crwn23 mutants apparently rules out a role for NMCP proteins in blue light-induced nuclear movement (Sakamoto and Takagi, 2013), although protein complementation cannot be completely discarded.

As indicated above, NMCPs show many analogies to lamins (Ciska and Moreno Díaz de la Espina, 2013) and in the future, even more may emerge as our understanding of these proteins improves. For all these reasons, and also because they are conserved in all land plants and they localize in the lamina (Masuda et al., 1993, 1997; Dittmer et al., 2007; Ciska et al., 2013; Sakamoto and Takagi, 2013), NMCPs are considered to be the plant analogs of lamins and the main components of the filament mesh that constitutes the plant lamina.

\section{OTHER COMPONENTS OF THE PLANT LAMINA}

While the plant lamina and its main structural components, the NMCP proteins, have now been relatively well characterized, the proteins anchoring this structure to the INM, NPCs, chromatin and the cytoskeleton remain largely unknown. Plants lack orthologs of the metazoan lamin-interacting proteins that attach the lamina to the INM, such as the LBR (lamin B receptor), LEM domain proteins and nesprins, or to NPCs, such as Nup153 (Mans et al., 2004). Hence, it would appear likely that they have evolved specific NMCP-interacting proteins that anchor the lamina to the NE and NPCs, also participating in the attachment of chromatin, and in the control of other nuclear and cellular activities regulated by lamins in metazoans. Searching for the partners of NMCPs in the lamina is fundamental to understand the functions and organization of this structure, yet to date, only one NMCP binding protein has been unequivocally identified (Graumann, 2014), even though the functional analysis of mutants suggests that direct or indirect interactions could occur with several proteins in the NPCs and NE, such as Nup136 (Tamura and Hara-Nishimura, 2011), SUN proteins (Graumann et al., 2010; Oda and Fukuda, 2011; Zhou et al., 2012; Graumann, 2014); WIPs (Zhou et al., 2012) and WITs (Tamura et al., 2013; Figure 4).

\section{CONNECTION OF THE PLANT LAMINA WITH THE NPCs}

As occurs in metazoa (Aaronson and Blobel, 1975) and protozoa (Rout and Field, 2001), NPCs associate with the isolated plant lamina (Moreno Diaz de la Espina, 2009). Moreover, feSEM analysis of the plant NE suggests that a direct interaction may occur between the filaments of the plant lamina and the inner ring of the NPCs (Figure 2C; Fiserova et al., 2009). However, the proteins that mediate this interaction are yet to be defined. In vertebrates, Nup153 is a mobile nucleoporin that is located in the inner ring of the NPCs and it associates with the Ig fold of both type A and B lamins (Al-Haboubi et al., 2011). Thus, Nup153 is believed to be involved in the interaction between the lamina and NPCs (Smythe et al., 2000; Walther et al., 2001), although other proteins may also participate in this interaction. Plants have a functional homolog of Nup153, Nup136 that was proposed to link the NPCs to the plant lamina (Tamura and Hara-Nishimura, 2011, 2013). Nup136 is unique to higher plants and while it shares no sequence similarity with vertebrate Nup153, it has some characteristics in common with the latter and it is also mobile, as demonstrated by FRAP analysis (Tamura et al., 2010). Although a direct interaction of NMCPs with Nup136 has not been proved, over-expression and down-regulation experiments showed that Nup136 and linc/crwn mutants have similar morphological alterations in the nucleus (Dittmer et al., 2007; Tamura et al., 2010; Sakamoto and Takagi, 2013), suggesting that both proteins interact and regulate nuclear morphology, and that Nup136 also links the NPC to the lamina (Tamura and Hara-Nishimura, 2011). It has been speculated that nuclear pore anchor (NUA), a nucleoporin of the nuclear pore basket, is involved in establishing nuclear architecture (Xu et al., 2007a,b). NUA is the plant homolog of the vertebrate nucleoporin Tpr, a long coiled coil protein that constitutes the scaffold of the nuclear pore basket (Strambio-De-Castillia et al., 2010). NUA accumulates in the inner side of the NE but its interaction with NMCP proteins and its role in nuclear organization have yet to be defined.

\section{THE LAMINA AND NUCLEOCYTOPLASMIC BRIDGING COMPLEXES IN PLANTS}

Amongst the integral proteins of the INM that bind to lamins in the metazoan lamina are the SUN domain proteins, which interact with the KASH domain proteins in the perinuclear space of the ONM to form the LINC complexes. The latter constitute the core of the connection between the nuclear lamina and the perinuclear cytoskeleton, forming a nucleocytoplasmic continuum. The LINC complexes fulfill a mechanical role in nuclear positioning and movement, centrosome attachment to the ONM, linking the nucleoskeleton to the cytoskeleton, and telomere positioning during meiosis, as well as participating in non-mechanical events regulating nuclear shape and size, and acting as specialized NE receptors (Tzur et al., 2006; Razafsky and Hodzic, 2009; Starr and Fridolfsson, 2010; Rothballer and Kutay, 2013; Sosa et al., 2013; Tapley and Starr, 2013; Stewart and Burke, 2014).

SUN proteins are highly conserved in eukaryotes, and while single cell eukaryotes have one SUN protein, C. elegans and Drosophila have two, and mammals and plants have multiple SUN proteins expressed at different times during development. The two major mammalian SUN proteins, SUN1 and SUN2, are widely 
expressed, while SUN3, 4, and 5 expression is restricted to the testis (Starr and Fridolfsson, 2010; Sosa et al., 2013; Zhou and Meier, 2013). Metazoan SUN proteins have a conserved domain layout, with a nucleoplasmic N-terminus that interacts directly with the Ig fold of lamins, followed by a transmembrane domain, a predicted coiled coil segment that localizes to the perinuclear space and allows trimerization, and a C-terminal SUN domain of about 175 amino acids that interacts with the KASH domain of KASH proteins (Tzur et al., 2006; Sosa et al., 2012). KASH domain proteins are more diverse. Yeast and Drosophila have two KASH proteins, C. elegans has three and mammals have six such proteins, called nesprins (Zhou and Meier, 2013). KASH proteins have an N-terminal cytoplasmic segment of varying size, structure and function, and a conserved C-terminal KASH domain that includes a transmembrane domain and a luminal domain of 20-30 amino acids. A PPPX motif can be found at the end of the C-terminus of typical KASH proteins, with conserved hydrophobic residues that lie upstream of it, both features that are essential for interactions with KASH and SUN domains (Rothballer and Kutay, 2013). In the formation of metazoan LINC complexes, the SUN proteins in the INM form homotrimers through the association of their coiled coil domains. The three adjacent SUN domains form clover-like trimers that interact with the KASH domains of three independent proteins anchored to the ONM, and binding is further stabilized by the formation of an intermolecular disulphide bond that covalently links the SUN and KASH domains. The cytoplasmic domains of KASH proteins anchored in the ONM interact with microtubule motors or actin filaments at the nuclear surface in order to move nuclei or to generate forces at the NE (Starr and Fridolfsson, 2010; Sosa et al., 2012, 2013; Tapley and Starr, 2013).

Plants encode up to five different SUN domain proteins that can be categorized into two classes: the canonical C-terminal SUN proteins SUN1 and SUN2 that are the structural homologs of the animal and yeast SUN1 and SUN2 proteins, and that contain a conserved domain layout with a NLS in the N-terminal domain, a transmembrane domain, a coiled coil domain and a highly conserved C-terminal SUN domain (Graumann etal., 2010, 2013; Murphy et al., 2010; Oda and Fukuda, 2011); and the plant prevalent mid-SUN3 proteins that contain three transmembrane domains, one at the $\mathrm{N}$ - and two at the $\mathrm{C}$ - terminus, as well as a SUN domain in the middle of the protein, which is followed by a highly conserved PAD (PM3-associated) domain of unknown function and a coiled coil domain (Murphy et al., 2010; Graumann et al., 2013). Unlike C-terminal SUNs, mid-SUN proteins have not yet been physiologically investigated. AtSUN1 and AtSUN2 are highly immobile intrinsic components of the NE, as demonstrated by FRAP analysis (Graumann et al., 2010). Moreover, recent results indicate that they interact with NMCP proteins (Graumann, 2014), suggesting an association with the lamina. They also form homomers and heteromers in vivo through the interaction of their coiled coil domains, as demonstrated by FRET (Graumann et al., 2010), indicating that they may function as multimer complexes. The predicted 3D structure of the SUN domain of AtSUN1 revealed that the essential structures and amino acids involved in KASH binding are conserved in relation to HsSUN2, but not the residues dispensable for the SUN-KASH interaction
(Zhou and Meier, 2013). AtSUN1 and AtSUN2 are involved in regulating nuclear shape (Oda and Fukuda, 2011; Zhou et al., 2012), anchoring protein complexes to the NE (Zhou et al., 2012), and linking the nucleus to cytoskeleton (Tamura et al., 2013). Hence, like their animal counterparts, plant SUN proteins appear to be key components involved in different protein networks, including the lamina, NE and nucleocytoplasmic bridging complexes.

Despite the conservation of SUN proteins, plants do not contain homologs of the opisthokont KASH proteins, although novel plant-specific SUN-interacting proteins were identified in Arabidopsis, WIPs (tryptophan-proline-proline [WPP] domain interacting proteins). WIPs are ONM anchored proteins with a cytoplasmic coiled coil domain, a transmembrane domain and a C-terminal tail in the perinuclear space, and they have a terminal conserved VPT motif that is essential for the interaction with SUN proteins (Zhou et al., 2012). Arabidopsis has three WIP homologs AtWIP1, AtWIP2, and AtWIP3 that interact with SUN proteins through the SUN domain. Accordingly, the SUN-WIP bridge would be the plant counterpart of the SUN-KASH bridge that forms the metazoan LINC complex. WIP proteins also redundantly anchor the RanGAP (Ran GTPase activating protein) to the NE through an interaction involving the N-terminal specific WPP domain of RanGAP and the coiled coil domain of WIPs. In this way, the SUN-WIP interaction provides a NE bridging complex and the anchoring of RanGAP to this structure suggests additional functions for these complexes (Figure 4; Zhou et al., 2012; Zhou and Meier, 2013). The possibility that the SUN-WIP bridges could connect with the cytoskeleton was recently enhanced with the discovery of a new type of plant nucleocytoplasmic linker involved in the regulation of nuclear shape and movement. This linker consists of a plant-specific myosin motor (Myosin Xl-i) that binds to both the actin filaments of the perinuclear cytoskeleton and the ONM WIT (WPP domain interacting tail anchored) proteins, with a similar domain organization to WIPs (Zhao et al., 2008), and which in turn interacts with the SUN-WIP bridge (Figure 4; Tamura et al., 2013).

Hence, NE bridging complexes connected to the lamina exist in plants. In this regard, while the INM components of these complexes are conserved in plants, the SUN proteins, their ONM partners are plant-specific and share no similarity with the KASH proteins. Thus, while the linkers of the nucleoskeleton to cytoskeleton (LINC) complexes are conserved in animals, they appear to have partially diverged in plants. The reported plant LINC complexes are involved in connecting the nucleus with actin filaments through a myosin motor that interacts with a plant-specific ONM protein, a mechanism that is unique to plants (Tamura et al., 2013). They are also implicated in the control of nuclear shape and movement in response to environmental stimuli, yet not in light-induced nuclear movement (Tamura et al., 2013). Hence, different mechanisms driving nuclear movement apparently exist in plants. As indicated above, plant LINC complexes also perform other unique functions, such as the anchoring of RanGAP to the ONM (Zhou et al., 2012).

\section{PERSPECTIVES}

Significant advances have been made in recent years in terms of the structural characterization of the plant lamina (Fiserova 


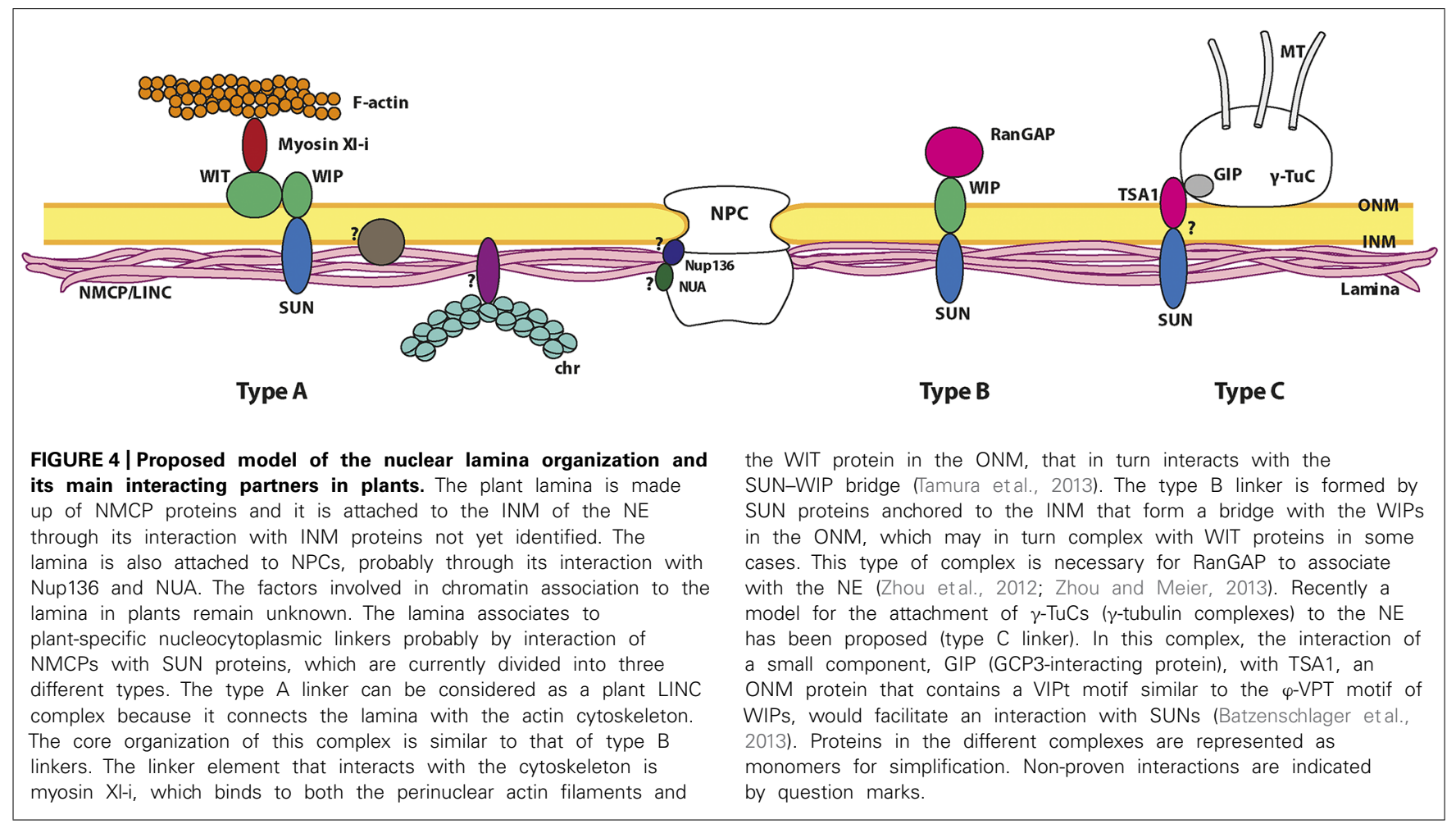

et al., 2009), as well as in the identification of its major protein components, the NMCP proteins considered to be functional analogs of metazoan lamins (Ciska and Moreno Díaz de la Espina, 2013). Nevertheless, considerable work is still needed, not only to advance in the knowledge of the mechanisms of assembly of NMCP proteins in the lamina and their functions but also, to fully understand the complete protein composition of this structure, the interactions therein and the roles it fulfills. One of the issues pending is the determination of the set of plant-specific proteins that drive the association of the lamina with the INM, NPCs, nucleocytoplasmic bridging complexes and chromatin, and the molecular interactions responsible for the association of these structures with NMCPs. The characterization of these proteins would represent an important advance in our understanding of the composition of the plant lamina and the protein interactions therein.

The bridging complexes that connect the plant lamina with the cytoskeleton are now beginning to be characterized. Their INM components (SUN proteins) are conserved, while those associated with the ONM are plant-specific (WIP proteins), evidence that the eukaryotic LINC complexes have partially diverged (Zhou et al., 2012; Zhou and Meier, 2013). The plant SUN-WIP core complexes are involved in connecting the nucleoskeleton with the actin cytoskeleton through a mechanism other than that involving animal LINC complexes. This interaction involves a plant-specific myosin motor that interacts with both actin filaments and a WIT protein, the latter associating with the WIP core protein of the complex (Tamura et al., 2013). The SUN-WIP complexes are also involved in anchoring protein complexes to the NE, like RanGAP, which fulfills plant-specific functions (Zhou et al., 2012). Apart from the association of NMCPs and SUNs (Graumann, 2014) the mechanisms that are responsible for stabilizing these complexes in the lamina remain unknown. The $\gamma$-Tubulin complexes $(\gamma$-TuCs) that nucleate MTs at the ONM are speculated to associate through the interaction of the small protein components of these complexes, GIPs (GCP3-interacting proteins). GIPs are required for correct $\gamma$-TuC localization at the NE, partnering TSA1 (TonSoKu [TSK]-associating protein 1), which has been proposed to interact with SUNs in the perinuclear space through its VIPT motif (Figure 4; Batzenschlager et al., 2013). Hence, the nucleocytoplasmic linker involved in the association of these complexes appears to display a quite diverse composition.

Despite the advances in our understanding of the plant lamina in the last few years, we still have very limited information about this NE component, and there are still many questions to be answered regarding the composition and functions of this structure. Which proteins link the NMCP-based lamina to the INM, nucleocytoplasmic linkers and chromatin? How is the plant lamina involved in chromatin tethering, organization and regulation? What are the functional capacities of the plant lamina? The study of the plant lamina is a field with great potential in plant nuclear biology, which will shed light on the mechanisms regulating nuclear shape and architecture, the connection of the nucleoskeleton to the cytoskeleton, nuclear positioning and movement, chromosome organization and positioning, gene expression, etc.

\section{ACKNOWLEDGMENTS}

We are grateful to Drs Jindriska Fiserova and Martin W. Goldberg for kindly providing feSEM Figures 2B,C, Enrique García Isidoro for help with Figure 4 and Dr. Mark Sefton for editorial 
assistance. We acknowledge the support from the Spanish Ministry of Science and Innovation [BFU2010-15900] and the CSIC [PIE 201020E019]. Malgorzata Ciska was supported by a grant from the Junta de Ampliación de Estudios (JAEPre_08_00012/JAEPre027) and by PIE $201020 \mathrm{E} 019$.

\section{REFERENCES}

Aaronson, R. P., and Blobel, G. (1975). Isolation of nuclear pore complexes in association with a lamina. Proc. Natl. Acad. Sci. U.S.A. 72, 1007-1011. doi: 10.1073/pnas.72.3.1007

Aebi, U., Cohn, J., Buhle, L., and Gerace, L. (1986). The nuclear lamina is a meshwork of intermediate-type filaments. Nature 323, 560-564. doi: 10.1038/323 $560 \mathrm{a} 0$

Al-Haboubi, T., Shumaker, D. K., Koser, J., Wehnert, M., and Fahrenkrog, B. (2011). Distinct association of the nuclear pore protein Nup153 with A- and B-type lamins. Nucleus 2, 500-509. doi: 10.4161/nucl.2.5.17913

Batsios, P., Peter, T., Baumann, O., Stick, R., Meyer, I., and Graf, R. (2012). A lamin in lower eukaryotes? Nucleus 3, 237-243. doi: 10.4161/nucl.20149

Batzenschlager, M., Masoud, K., Janski, N., Houlne, G., Herzog, E., Evrard, J. L., et al. (2013). The GIP gamma-tubulin complex-associated proteins are involved in nuclear architecture in Arabidopsis thaliana. Front. Plant Sci. 4:480. doi: 10.3389/fpls.2013.00480

Beams, H. W., Tahmisian, T. N., Devine, R., and Anderson, E. (1957). Ultrastructure of the nuclear membrane of a gregarine parasitic in grasshoppers. Exp. Cell Res. 13, 200-204.

Benavente, R., Krohne, G., and Franke, W. W. (1985). Cell type-specific expression of nuclear lamina proteins during development of Xenopus laevis. Cell 41, 177-190. doi: 10.1016/0092-8674(85)90072-8

Ben-Harush, K., Wiesel, N., Frenkiel-Krispin, D., Moeller, D., Soreq, E., Aebi, U., et al. (2009). The supramolecular organization of the C. elegans nuclear lamin filament. J. Mol. Biol. 386, 1392-1402. doi: 10.1016/j.jmb.2008.12.024

Blumenthal, S. S., Clark, G. B., and Roux, S. J. (2004). Biochemical and immunological characterization of pea nuclear intermediate filament proteins. Planta 218 , 965-975. doi: 10.1007/s00425-003-1182-5

Broers, J. L., Machiels, B. M., Kuijpers, H. J., Smedts, F., Van Den Kieboom, R., Raymond, Y., et al. (1997). A- and B-type lamins are differentially expressed in normal human tissues. Histochem. Cell Biol. 107, 505-517. doi: 10.1007/s004180050138

Burke, B., and Stewart, C. L. (2013). The nuclear lamins: flexibility in function. Nat. Rev. Mol. Cell Biol. 14, 13-24. doi: 10.1038/nrm3488

Butin-Israeli, V., Adam, S. A., Goldman, A. E., and Goldman, R. D. (2012). Nuclear lamin functions and disease. Trends Genet. 28, 464-471. doi: 10.1016/j.tig.2012.06.001

Ciska, M., Masuda, K., and Moreno Diaz De La Espina, S. (2013). Lamin-like analogues in plants: the characterization of NMCP1 in Allium cepa. J. Exp. Bot. 64, 1553-1564. doi: 10.1093/jxb/ert020

Ciska, M., and Moreno Díaz de la Espina, S. (2013). NMCP/LINC proteins: putative lamin analogs in plants? Plant Signal. Behav. 8:e26669.

Crisp, M., Liu, Q., Roux, K., Rattner, J. B., Shanahan, C., Burke, B., et al. (2006). Coupling of the nucleus and cytoplasm: role of the LINC complex. J. Cell Biol. 172, 41-53. doi: 10.1083/jcb.200509124

Chen, B., Cai, S. T., and Zhai, Z. H. (1994). [Investigation of nuclear lamina in Tetrahymena thermophila]. Shi Yan Sheng Wu Xue Bao 27, 153-163.

Davidson, P. M., and Lammerding, J. (2013). Broken nuclei - lamins, nuclear mechanics, and disease. Trends Cell Biol. 24, 247-256. doi: 10.1016/j.tcb.2013.11.004

Dechat, T., Adam, S. A., and Goldman, R. D. (2009). Nuclear lamins and chromatin: when structure meets function. Adv. Enzyme Regul. 49, 157-166. doi: 10.1016/j.advenzreg.2008.12.003

Dechat, T., Adam, S. A., Taimen, P., Shimi, T., and Goldman, R. D. (2010). Nuclear lamins. Cold Spring Harb. Perspect. Biol. 2:a000547. doi: 10.1101/cshperspect.a000547

Dechat, T., Pfleghaar, K., Sengupta, K., Shimi, T., Shumaker, D. K., Solimando, L., et al. (2008). Nuclear lamins: major factors in the structural organization and function of the nucleus and chromatin. Genes Dev. 22, 832-853. doi: 10.1101/gad. 1652708

Dittmer, T. A., and Misteli, T. (2011). The lamin protein family. Genome Biol. 12:222. doi: 10.1186/gb-2011-12-5-222
Dittmer, T. A., and Richards, E. J. (2008). Role of LINC proteins in plant nuclear morphology. Plant Signal. Behav. 3, 485-487. doi: 10.4161/psb.3.7.5682

Dittmer, T. A., Stacey, N. J., Sugimoto-Shirasu, K., and Richards, E. J. (2007). LITTLE NUCLEI genes affecting nuclear morphology in Arabidopsis thaliana. Plant Cell 19, 2793-2803. doi: 10.1105/tpc.107.053231

Dubois, K. N., Alsford, S., Holden, J. M., Buisson, J., Swiderski, M., Bart, J. M., et al. (2012). NUP-1 is a large coiled-coil nucleoskeletal protein in trypanosomes with lamin-like functions. PLoS Biol. 10:e1001287. doi: 10.1371/journal.pbio.1001287 Dwyer, N., and Blobel, G. (1976). A modified procedure for the isolation of a pore complex-lamina fraction from rat liver nuclei. J. Cell Biol. 70, 581-591. doi: $10.1083 /$ jcb.70.3.581

Fawcett, D. W. (1966). On the occurrence of a fibrous lamina on the inner aspect of the nuclear envelope in certain cells of vertebrates. Am. J. Anat. 119, 129-145. doi: 10.1002/aja.1001190108

Field, M. C., Horn, D., Alsford, S., Koreny, L., and Rout, M. P. (2012). Telomeres, tethers and trypanosomes. Nucleus 3, 478-486. doi: 10.4161/nucl.22167

Fiserova, J., and Goldberg, M. W. (2010). Relationships at the nuclear envelope: lamins and nuclear pore complexes in animals and plants. Biochem. Soc. Trans. 38, 829-831. doi: 10.1042/BST0380829

Fiserova, J., Kiseleva, E., and Goldberg, M. W. (2009). Nuclear envelope and nuclear pore complex structure and organization in tobacco BY-2 cells. Plant J. 59, 243255. doi: 10.1111/j.1365-313X.2009.03865.x

Frajola, W. J., Greider, M. H., and Kostir, W. J. (1956). Electron microscopy of the nuclear membrane of Amoeba proteus. J. Biophys. Biochem. Cytol. 2, 445-448.

Franke, W. W. (1987). Nuclear lamins and cytoplasmic intermediate filament proteins: a growing multigene family. Cell 48, 3-4. doi: 10.1016/00928674(87)90345-X

Gangemi, F., and Degano, M. (2013). Disease-associated mutations in the coil 2B domain of human lamin $\mathrm{A} / \mathrm{C}$ affect structural properties that mediate dimerization and intermediate filament formation. J. Struct. Biol. 181, 17-28. doi: 10.1016/j.jsb.2012.10.016

Gerace, L., and Blobel, G. (1980). The nuclear envelope lamina is reversibly depolymerized during mitosis. Cell 19, 277-287. doi: 10.1016/0092-8674(80)90409-2

Gerace, L., Blum, A., and Blobel, G. (1978). Immunocytochemical localization of the major polypeptides of the nuclear pore complex-lamina fraction. Interphase and mitotic distribution. J. Cell Biol. 79, 546-566. doi: 10.1083/jcb.79. 2.546

Gerace, L., and Huber, M. D. (2012). Nuclear lamina at the crossroads of the cytoplasm and nucleus. J. Struct. Biol. 177, 24-31. doi: 10.1016/j.jsb.2011.11.007

Goldberg, M. W., Fiserova, J., Huttenlauch, I., and Stick, R. (2008a). A new model for nuclear lamina organization. Biochem. Soc. Trans. 36, 1339-1343. doi: 10.1042/BST0361339

Goldberg, M. W., Huttenlauch, I., Hutchison, C. J., and Stick, R. (2008b). Filaments made from A- and B-type lamins differ in structure and organization. J. Cell Sci. 121, 215-225. doi: 10.1242/jcs.022020

Graumann, K. (2014). Evidence for LINC1-SUN associations at the plant nuclear periphery. PLoS ONE 9:e93406. doi: 10.1371/journal.pone.0093406

Graumann, K., Bass, H. W., and Parry, G. (2013). SUNrises on the international plant nucleus consortium: SEB Salzburg 2012. Nucleus 4, 3-7. doi: 10.4161/nucl.23385

Graumann, K., Runions, J., and Evans, D. E. (2010). Characterization of SUNdomain proteins at the higher plant nuclear envelope. Plant J. 61, 134-144. doi: 10.1111/j.1365-313X.2009.04038.x

Ho, C. Y., and Lammerding, J. (2012). Lamins at a glance. J. Cell Sci. 125, 2087-2093. doi: $10.1242 /$ jcs.087288

Kapinos, L. E., Schumacher, J., Mucke, N., Machaidze, G., Burkhard, P., Aebi, U., et al. (2010). Characterization of the head-to-tail overlap complexes formed by human lamin A, B1 and B2 "half-minilamin" dimers. J. Mol. Biol. 396, 719-731. doi: 10.1016/j.jmb.2009.12.001

Kimura, Y., Fujino, K., Ogawa, K., and Masuda, K. (2014). Localization of Daucus carota NMCP1 to the nuclear periphery: the role of the N-terminal region and an NLS-linked sequence motif, RYNLRR, in the tail domain. Front. Plant Sci. 5:62. doi: 10.3389/fpls.2014.00062

Kimura, Y., Kuroda, C., and Masuda, K. (2010). Differential nuclear envelope assembly at the end of mitosis in suspension-cultured Apium graveolens cells. Chromosoma 119, 195-204. doi: 10.1007/s00412-009-0248-y

Kolb, T., Maass, K., Hergt, M., Aebi, U., and Herrmann, H. (2011). Lamin A and lamin $\mathrm{C}$ form homodimers and coexist in higher complex forms both in the nucleoplasmic fraction and in the lamina of cultured human cells. Nucleus 2, 425-433. doi: 10.4161/nucl.2.5.17765 
Kruger, A., Batsios, P., Baumann, O., Luckert, E., Schwarz, H., Stick, R., et al. (2012). Characterization of NE81, the first lamin-like nucleoskeleton protein in a unicellular organism. Mol. Biol. Cell 23, 360-370. doi: 10.1091/mbc.E11-07-0595

Lang, S., and Loidl, P. (1993). Identification of proteins immunologically related to vertebrate lamins in the nuclear matrix of the myxomycete Physarum polycephalum. Eur. J. Cell Biol. 61, 177-183.

Li, H., and Roux, S. J. (1992). Casein kinase II protein kinase is bound to laminamatrix and phosphorylates lamin-like protein in isolated pea nuclei. Proc. Natl. Acad. Sci. U.S.A. 89, 8434-8438. doi: 10.1073/pnas.89.18.8434

Mans, B. J., Anantharaman, V., Aravind, L., and Koonin, E. V. (2004). Comparative genomics, evolution and origins of the nuclear envelope and nuclear pore complex. Cell Cycle 3, 1612-1637. doi: 10.4161/cc.3.12.1316

Masuda, K., Haruyama, S., and Fujino, K. (1999). Assembly and disassembly of the peripheral architecture of the plant cell nucleus during mitosis. Planta 210, 165-167. doi: 10.1007/s004250050666

Masuda, K., Takahashi, S., Nomura, K., Arimoto, M., and Inoue, M. (1993). Residual structure and constituent proteins of the peripheral framework of the cell nucleus in somatic embryos from Daucus carota L. Planta 191, 532-540. doi: 10.1007/BF00195755

Masuda, K., Xu, Z. J., Takahashi, S., Ito, A., Ono, M., Nomura, K., et al. (1997). Peripheral framework of carrot cell nucleus contains a novel protein predicted to exhibit a long alpha-helical domain. Exp. Cell Res. 232, 173-181. doi: 10.1006/excr.1997.3531

Mckeon, F. D., Kirschner, M. W., and Caput, D. (1986). Homologies in both primary and secondary structure between nuclear envelope and intermediate filament proteins. Nature 319, 463-468. doi: 10.1038/319463a0

Melcer, S., Gruenbaum, Y., and Krohne, G. (2007). Invertebrate lamins. Exp. Cell Res. 313, 2157-2166. doi: 10.1016/j.yexcr.2007.03.004

Minguez, A., Franca, S., and Moreno Diaz De La Espina, S. (1994). Dinoflagellates have a eukaryotic nuclear matrix with lamin-like proteins and topoisomerase II. J. Cell Sci. 107, 2861-2873.

Minguez, A., and Moreno Diaz de la Espina, S. (1993). Immunological characterization of lamins in the nuclear matrix of onion cells. J. Cell Sci. 106 431-439.

Moir, R. D., Yoon, M., Khuon, S., and Goldman, R. D. (2000). Nuclear lamins A and B1: different pathways of assembly during nuclear envelope formation in living cells. J. Cell Biol. 151, 1155-1168. doi: 10.1083/jcb.151.6.1155

Moreno Diaz de la Espina, S. (1995). Nuclear matrix isolated from plant cells. Int. Rev. Cytol. 162B, 75-139.

Moreno Diaz de la Espina, S. (2009). “The plant nucleoskeleton," in Functional Organization of the Plant Nucleus, ed. I. Meier (Berlin Heidelberg: Springer), 79-100. doi: 10.1007/978-3-540-71058-5_5

Moreno Diaz de la Espina, S., Barthellemy, I., and Cerezuela, M. A. (1991). Isolation and ultrastructural characterization of the residual nuclear matrix in a plant cel system. Chromosoma 100, 110-117. doi: 10.1007/BF00418244

Moriguchi, K., Suzuki, T., Ito, Y., Yamazaki, Y., Niwa, Y., and Kurata, N. (2005). Functional isolation of novel nuclear proteins showing a variety of subnuclear localizations. Plant Cell 17, 389-403. doi: 10.1105/tpc.104. 028456

Murphy, S. P., Simmons, C. R., and Bass, H. W. (2010). Structure and expression of the maize (Zea mays L.) SUN-domain protein gene family: evidence for the existence of two divergent classes of SUN proteins in plants. BMC Plant Biol. 10:269. doi: 10.1186/1471-2229-10-269

Oda, Y., and Fukuda, H. (2011). Dynamics of Arabidopsis SUN proteins during mitosis and their involvement in nuclear shaping. Plant J. 66, 629-641. doi: 10.1111/j.1365-313X.2011.04523.x

Ostlund, C., Sullivan, T., Stewart, C. L., and Worman, H. J. (2006). Dependence of diffusional mobility of integral inner nuclear membrane proteins on A-type lamins. Biochemistry 45, 1374-1382. doi: 10.1021/bi052156n

Pappas, G. D. (1956). The fine structure of the nuclear envelope of Amoeba proteus. J. Biophys. Biochem. Cytol. 2, 431-434. doi: 10.1083/jcb.2.4.431

Peter, A., and Stick, R. (2012). Evolution of the lamin protein family: what introns can tell. Nucleus 3, 44-59. doi: 10.4161/nucl.18927

Razafsky, D., and Hodzic, D. (2009). Bringing KASH under the SUN: the many faces of nucleo-cytoskeletal connections. J. Cell Biol. 186, 461-472. doi: 10.1083/jcb.200906068

Rose, A., Manikantan, S., Schraegle, S. J., Maloy, M. A., Stahlberg, E. A., and Meier, I. (2004). Genome-wide identification of Arabidopsis coiled-coil proteins and establishment of the ARABI-COIL database. Plant Physiol. 134, 927-939. doi: 10.1104/pp.103.035626

Rothballer, A., and Kutay, U. (2013). The diverse functional LINCs of the nuclear envelope to the cytoskeleton and chromatin. Chromosoma 122, 415-429. doi: 10.1007/s00412-013-0417-x

Rout, M. P., and Field, M. C. (2001). Isolation and characterization of subnuclear compartments from Trypanosoma brucei. Identification of a major repetitive nuclear lamina component. J. Biol. Chem. 276, 38261-38271. doi: 10.1074/jbc.M104024200

Rudzinska, M. A. (1956). Further observations on the fine structure of the macronucleus in Tokophrya infusionum. J. Biophys. Biochem. Cytol. 2, 425-430. doi: 10.1083/jcb.2.4.425

Sakamoto, Y., and Takagi, S. (2013). LITTLE NUCLEI 1 and 4 regulate nuclear morphology in Arabidopsis thaliana. Plant Cell Physiol. 54, 622-633. doi: $10.1093 / \mathrm{pcp} / \mathrm{pct} 031$

Scheer, U., Kartenbeck, J., Trendelenburg, M. F., Stadler, J., and Franke, W. W. (1976). Experimental disintegration of the nuclear envelope. Evidence for poreconnecting fibrils. J. Cell Biol. 69, 1-18. doi: 10.1083/jcb.69.1.1

Schmidt, M., Grossmann, U., and Krohne, G. (1995). The nuclear membraneassociated honeycomb structure of the unicellular organism Amoeba proteus: on the search for homologies with the nuclear lamina of metazoa. Eur. J. Cell Biol. 67, 199-208.

Simon, D. N., and Wilson, K. L. (2011). The nucleoskeleton as a genome-associated dynamic 'network of networks.' Nat. Rev. Mol. Cell Biol. 12, 695-708. doi: $10.1038 / \mathrm{nrm} 3207$

Simon, D. N., and Wilson, K. L. (2013). Partners and post-translational modifications of nuclear lamins. Chromosoma 122, 13-31. doi: 10.1007/s00412-0130399-8

Smythe, C., Jenkins, H. E., and Hutchison, C. J. (2000). Incorporation of the nuclear pore basket protein nup153 into nuclear pore structures is dependent upon lamina assembly: evidence from cell-free extracts of Xenopus eggs. EMBO J. 19, 39183931. doi: 10.1093/emboj/19.15.3918

Sosa, B. A., Kutay, U., and Schwartz, T. U. (2013). Structural insights into LINC complexes. Curr. Opin. Struct. Biol. 23, 285-291. doi: 10.1016/j.sbi.2013.03.005

Sosa, B. A., Rothballer, A., Kutay, U., and Schwartz, T. U. (2012). LINC complexes form by binding of three KASH peptides to domain interfaces of trimeric SUN proteins. Cell 149, 1035-1047. doi: 10.1016/j.cell.2012.03.046

Starr, D. A., and Fridolfsson, H. N. (2010). Interactions between nuclei and the cytoskeleton are mediated by SUN-KASH nuclear-envelope bridges. Annu. Rev. Cell Dev. Biol. 26, 421-444. doi: 10.1146/annurev-cellbio-100109-104037

Stewart, C., and Burke, B. (2014). The missing LINC: a mammalian KASH-domain protein coupling meiotic chromosomes to the cytoskeleton. Nucleus 5. doi: 10.4161/nucl.27819 [Epub ahead of print].

Strambio-De-Castillia, C., Niepel, M., and Rout, M. P. (2010). The nuclear pore complex: bridging nuclear transport and gene regulation. Nat. Rev. Mol. Cell Biol. 11, 490-501. doi: 10.1038/nrm2928

Tamura, K., Fukao, Y., Iwamoto, M., Haraguchi, T., and Hara-Nishimura, I. (2010). Identification and characterization of nuclear pore complex components in Arabidopsis thaliana. Plant Cell 22, 4084-4097. doi: 10.1105/tpc.110. 079947

Tamura, K., and Hara-Nishimura, I. (2011). Involvement of the nuclear pore complex in morphology of the plant nucleus. Nucleus 2, 168-172. doi: 10.4161/nucl.2.3.16175

Tamura, K., and Hara-Nishimura, I. (2013). The molecular architecture of the plant nuclear pore complex. J. Exp. Bot. 64, 823-832. doi: 10.1093/jxb/ers258

Tamura, K., Iwabuchi, K., Fukao, Y., Kondo, M., Okamoto, K., Ueda, H., et al. (2013). Myosin XI-i links the nuclear membrane to the cytoskeleton to control nuclear movement and shape in Arabidopsis. Curr. Biol. 23, 1776-1781. doi: 10.1016/j.cub.2013.07.035

Tapley, E. C., and Starr, D. A. (2013). Connecting the nucleus to the cytoskeleton by SUN-KASH bridges across the nuclear envelope. Curr. Opin. Cell Biol 25, 57-62. doi: 10.1016/j.ceb.2012.10.014

Tzur, Y. B., Wilson, K. L., and Gruenbaum, Y. (2006). SUN-domain proteins: 'Velcro' that links the nucleoskeleton to the cytoskeleton. Nat. Rev. Mol. Cell Biol. 7, 782-788. doi: 10.1038/nrm2003.

van Zanten, M., Carles, A., Li, Y., and Soppe, W. J. (2012). Control and consequences of chromatin compaction during seed maturation in Arabidopsis thaliana. Plant Signal. Behav. 7, 338-341. doi: 10.4161/psb.19281 
van Zanten, M., Koini, M. A., Geyer, R., Liu, Y., Brambilla, V., Bartels, D., et al. (2011). Seed maturation in Arabidopsis thaliana is characterized by nuclear size reduction and increased chromatin condensation. Proc. Natl. Acad. Sci. U.S.A. 108, 20219-20224. doi: 10.1073/pnas.1117726108

Walther, T. C., Fornerod, M., Pickersgill, H., Goldberg, M., Allen, T. D., and Mattaj, I. W. (2001). The nucleoporin Nup153 is required for nuclear pore basket formation, nuclear pore complex anchoring and import of a subset of nuclear proteins. EMBO J. 20, 5703-5714. doi: 10.1093/emboj/20.20.5703

Wang, H., Dittmer, T. A., and Richards, E. J. (2013). Arabidopsis CROWDED NUCLEI (CRWN) proteins are required for nuclear size control and heterochromatin organization. BMC Plant Biol. 13:200. doi: 10.1186/1471-222913-200

Wilson, K. L., and Foisner, R. (2010). Lamin-binding Proteins. Cold Spring Harb. Perspect. Biol. 2:a000554. doi: 10.1101/cshperspect.a000554

Xu, X. M., Rose, A., and Meier, I. (2007a). NUA activities at the plant nuclear pore. Plant Signal. Behav. 2, 553-555. doi: 10.4161/psb.2.6.4836

Xu, X. M., Rose, A., Muthuswamy, S., Jeong, S. Y., Venkatakrishnan, S., Zhao, Q., et al. (2007b). NUCLEAR PORE ANCHOR, the Arabidopsis homolog of Tpr/Mlp1/Mlp2/megator, is involved in mRNA export and SUMO homeostasis and affects diverse aspects of plant development. Plant Cell 19, 1537-1548. doi: $10.1105 /$ tpc.106.049239

Zhao, Q., Brkljacic, J., and Meier, I. (2008). Two distinct interacting classes of nuclear envelope-associated coiled-coil proteins are required for the tissue-specific nuclear envelope targeting of Arabidopsis RanGAP. Plant Cell 20, 1639-1651. doi: $10.1105 /$ tpc. 108.059220

Zhou, X., Graumann, K., Evans, D. E., and Meier, I. (2012). Novel plant SUN-KASH bridges are involved in RanGAP anchoring and nuclear shape determination. J. Cell Biol. 196, 203-211. doi: 10.1083/jcb.201108098

Zhou, X., and Meier, I. (2013). How plants LINC the SUN to KASH. Nucleus 4, 206-215. doi: 10.4161/nucl.24088

Conflict of Interest Statement: The authors declare that the research was conducted in the absence of any commercial or financial relationships that could be construed as a potential conflict of interest.

Received: 03 February 2014; accepted: 08 April 2014; published online: 29 April 2014. Citation: Ciska M and Moreno Díaz de la Espina S (2014) The intriguing plant nuclear lamina. Front. Plant Sci. 5:166. doi: 10.3389/fpls.2014.00166

This article was submitted to Plant Cell Biology, a section of the journal Frontiers in Plant Science.

Copyright (c) 2014 Ciska and Moreno Díaz de la Espina. This is an open-access article distributed under the terms of the Creative Commons Attribution License (CC BY). The use, distribution or reproduction in other forums is permitted, provided the original author(s) or licensor are credited and that the original publication in this journal is cited, in accordance with accepted academic practice. No use, distribution or reproduction is permitted which does not comply with these terms. 